01

\title{
Low-Frequency Vibrational Modes in Small Polypeptides of Essential Amino Acids *
}

\author{
(C) Alireza Kokabi, Hamidreza Ghanbari Khorram, Zeinab Meivand, Tina Bayatian, Ehsan Ghorbani Rad \\ Department of Electrical Engineering, Hamedan University of Technology, \\ 65155 Hamedan, Iran \\ e-mail: alireza.kokabi@hut.ac.ir
}

Received October 06, 2018

Revised October 06, 2018

Accepted March 15, 2019

The length-dependent low-frequency terahertz absorption spectrum of the essential amino acid chains has been investigated. Since this special type of amino acids cannot be synthesized inside the human body, the feasibility of noninvasively monitoring their deficiency is of significant importance. Here, the considered chains consist of two to six identical essential amino acids forming homogeneous polypeptides. The terahertz vibrational modes are calculated for different chain sizes of these polypeptides. The low-frequency terahertz spectra show unique and specific absorption peaks. Several fascinating patterns for the chain-size-dependent position of the peaks are reported here that are feasible of being applied for their noninvasive detection. For instance, different shared and identical characteristics peaks are observed for the specific polypeptides which are independent of the chain-size. Furthermore, it is shown that the observed trends for the maximum populated peak in some of the essential polypeptides can be considered as a possible footprint for in the terahertz spectrum.

Key words: essential amino acid chains, terahertz spectrum.

DOI: $10.21883 /$ OS.2019.09.48192.292-18

* Полная версия статьи опубликована в английской версии журнала. 